\title{
XPS Study of Multilayer Multicomponent Films
}

\author{
Alexander V. Lubenchenko, ${ }^{\mathrm{a}, *}$, Alexander A. Batrakov ${ }^{\mathrm{a}}$, Alexey B. Pavolotsky ${ }^{\mathrm{b}}$, Olga I. Lubenchenko ${ }^{\mathrm{a}}$, \\ Dmitriy. A. Ivanov ${ }^{a}$ \\ ${ }^{a}$ National Research University MPEI, Krasnokazarmennaya, 14, Moscow 111250, Russia \\ ${ }^{b}$ Chalmers University of Technology, Gteborg, 41296, Sweden
}

\begin{abstract}
In the paper, we propose XPS-based quantitative method for depth profile analysis of chemical and phase composition of multi-component and multi-layer samples. The method includes: (1) new method for background subtraction accounting for depth non-uniformity of electron energy losses; (2) new method for photoelectron line decomposition into elementary peaks, which accounts for physical nature of the decomposition parameters; (3) joint solving of both background subtraction and photoelectron line decomposition problems; (4) criterion for assessing of line decomposition accuracy; (5) simple formula for layer thickness extraction for multi-element and multi-layer sample. We apply the developed method for analysis of multilayer niobium oxide and sub-oxide films before and in course of ion milling.
\end{abstract}

Keywords: XPS; Chemical and phase analysis; Depth profiling; XPS background subtracting; XPS line decomposition; Niobium oxide

\section{Introduction}

X-ray Photoelectron Spectroscopy (XPS) is one of the most demanded methods of the surface analysis [1]. XPS allows extracting elemental and phase composition of the analyzed surfaces. For that, after background subtraction, position of the peaks formed by inelastically and elastically scattered photoelectrons in the XPS spectrum is determined. Intensities of those peaks are interpreted into the relative concentrations of the elements. The standard method for interpretation of XPS data is based on the assumption about uniform distribution of the elements across the whole depth of analysis. In reality though sample surface is always nonuniform and can be represented as consisting of layers. Failure to account

\footnotetext{
${ }^{*}$ Corresponding author

Email address: LubenchenkoAV@mpei.ru (Alexander V. Lubenchenko)
}

for that leads to significant errors. For example, assume a sample has on its surface a layer of thickness $d_{1}$ of material other than the the rest of the sample. In such case, following the Bouguer law, intensity of the peaks formed by the photo electrons under that surface layer will be reduced by the factor of $\exp \left(-d_{1} / \lambda_{1}\right)$, where $\lambda_{1}$ is electron mean free path in that surface layer. In case of XPS with $\mathrm{Mg} / \mathrm{Al}$-anode X-ray source, electron mean free path is of the order of a few nanometers. Consequently, even as thin as $1 \mathrm{~nm}$ surface layer will noticeably misrepresent the concentrations calculated by the standard mean concentration method.

For analysis of multilayer samples, more sophisticated calculation approach is needed. In paper [2, relationships for elements' relative concentrations in a layer were derived. However, for that one needs to assume the layer model and solve nonlinear system of equation for extracting of layer thicknesses. Analytic solution of such system of equations is pre- 
sented in paper 3 . Using of the methods accounting for multilayer surface structure, one needs though to find each element's partial peak intensity. This problem in turn depends on the accurate solution of the following standard problems of XPS: (1) Subtraction of background due to multiple inelastically scattered photoelectrons; (2) Line decomposition into partial peaks of not scattered and elastically scattered photoelectrons. These problems are significantly interlinked.

In this paper, we present a new method for solving of the standard problems of XPS. This includes background subtraction accounting for inelastic scattering nonuniform by the depth, as well as line decomposition into partial peaks, which is based on the physical model for photoelectron peak forming. These two problems are solved together within the presented approach. Presented in this paper, account for sample nonuniformity by depth significantly increases analysis accuracy as compared with the standard approach.

Sample surface usually not only is multilayer, but each layer itself is multicomponent and often multiphase. Layer-by-layer interpretation of such samples' XPS spectra is a complex reverse problem with multiple unknown parameters. For correct solving of such a problem, we propose: (1) Method for background subtraction accounting for electron energy losses different in volume and at surface; (2) Parameters for background and line shape calculation fixed for the whole range of the spectrum; (3) Line shape parameters calculated based on the Handbook of X-Ray Photoelectron Spectroscopy [4] spectra for pure uniform surfaces; (4) Formulas for chemically bonded elements photoelectron inherent peak width; (5) Joint interpretation of different lines of the element within the same model. We also propose a criterion for judging the quality of line decomposition to choose between alternative solutions. We suggest that calculations of mean relative stoichiometric coefficients should give the same result, when based either on different lines intensities, or on partial intensities of the peaks of the line together with chemical shift (see (14).

We applied the presented method to analysis of chemical and phase composition of niobium oxide films. Interest to niobium thin films and its compounds is motivated by its superconducting properties and consequent application in cryoelectronic devices. Surface oxide films affect negatively on superconducting properties of niobium, particularly when used as thin film. There are a number of papers, e.g. [5, 6. 7, 8, 9], dedicated to XPS analysis of Nb oxide films. However, there are no known publications communicating results of XPS analysis of phase composition of surface niobium oxide films. The present paper attempts to fill this gap.

\section{Experimental Details}

In this paper, we studied niobium surface oxide films before and after ion profiling. Niobium was deposited on silicon substrate by magnetron sputtering of $99.95 \% \mathrm{Nb}$ in Ar with the help of Pfeiffer Vacuum SLS630G sputtering cluster tool. Thickness of sputtered $\mathrm{Nb}$ was $200 \mathrm{~nm}$. Immediately after unloading the samples from the deposition system, the samples were covered by oxidation protecting layer of spun photoresist. Right before loading the samples to the XPS analysis chamber, the protecting layer was removed in an ultrasonic bath filled with acetone followed by iso-propanol. This way, the total exposure time of the samples to atmospheric air was about 10 minutes.

XPS spectra were recorded with the help of Nanofab 25 (NT-MDT) electron-ion spectroscopy platform. The base pressure in the analysis chamber was at the level of $10^{-8} \mathrm{~Pa}$ provided by a titanium sublimation pump and an ion pump. The residual pressure and composition of vacuum were monitored by a Bayard-Alpert vacuum gauge and a secondary ion mass-spectrometer. The load-lock was pumped with a turbo-molecular pump. In the load-lock, it was possible to store a number of samples at the residual pressure of $10^{-6} \mathrm{~Pa}$. For XPS analysis, the X-ray source SPECS XR 50 with a dual Al/Mg anode was used providing $1486.6 \mathrm{eV}$ and $1253.6 \mathrm{eV}$ photons. The X-ray source was located at 54.7 degrees relative to the analyzer axis. For surface charge neutralizing, flood electron gun SPECS FG 15/40 was used.

Semispheric electrostatic energy analyzer SPECS Phoibos 225 allowed recording of spectra in a broad 
range of energies between 0 and $15 \mathrm{keV}$ in the angle range of \pm 15 degrees with an energy resolution of $0.6 \mathrm{eV}$ FWHM measured at $15 \mathrm{keV}$. Energy analyzer was calibrated with a use of reference $\mathrm{Cu}, \mathrm{Ag}$ and $\mathrm{Au}$ samples. Energy resolution of spectrometer at $\mathrm{Ag} 3 d_{5 / 2}$ line was found to be $0.78 \mathrm{eV}$ for nonmonochromatic $\mathrm{MgK} \alpha$. All survey spectra scans were taken at a pass energy of $80 \mathrm{eV}$. The detailed scans of strong lines were in most cases recorded as wide as needed just to encompass the peak(s) of interest and were obtained with a pass energy of $20 \mathrm{eV}$. All spectra were recorded with use of $\mathrm{Mg}$ anode of the X-ray source. The energy analyzer was operated in Fixed Analyzer Transmission (FAT) mode.

For cleaning and ion profiling of the samples, an ion source SPECS IQE 12/38 was used. The ion source had differential pumping and was fed with $99.9995 \%$ pure Ar. For ion profiling, the ion beam was scanning the $2.8 \times 4.0 \mathrm{~mm}^{2}$ area at the incidence angle of 50 degrees to the surface normal. Ion profiling included total 9 cycles of sputtering with recording of XPS spectra before sputtering and after each cycle. For sputtering cycles \#1 - \#6, each cycle was 20 minute long and beam energy and current were $0.5 \mathrm{keV}$ and $0.5 \mu \mathrm{A}$, respectively. For sputtering cycles \#7-\#9, each cycle was 40 minutes long and ion energy and beam current were $3.0 \mathrm{keV}$ and $1.5 \mu \mathrm{A}$, respectively. Results of the ion profiling studies are summarized in the Section 4

\section{Theory}

Photoelectron spectrum is formed by not scattered, elastically scattered and inelastically multiply scattered photoelectrons. Analysis of peaks formed by not scattered and elastically scattered electrons gives layer-by-layer chemical and phase composition information (line-shape analysis). In this case, contribution of inelastically scattered photoelectrons is considered as unwanted background. Consequently, accuracy of the analysis depends critically on the procedure of background subtraction. Hence, analysis of multilayer and multicomponent samples is significantly complicated, as peaks of elastically scattered electrons will overlap each other and the peaks of inelastically scattered electrons.
Identification of layer-by-layer chemical and phase surface composition includes sequential solving of the following three problems: (1) Subtraction of the background due to inelastically scattered electrons; (2) Decomposition of the spectrum into its constituent peaks (extracting of intensity and position of the peaks); (3) Extracting of layers thicknesses of the multilayer sample. The first and the second problems are largely related. Unknown parameters that affect calculation of both background, and elastically scattered photoelectron peaks should be defined jointly.

\subsection{Background Subtraction}

Background subtraction in XPS analysis, most commonly follows methods of Shirley [10] and Tougaard [11, which give relatively simple calculation algorithms. Shirley method is purely mathematical technique, which does not account for specifics of electron inelastic scattering in solid. Because of that, background subtraction accuracy is often unpredictable. Method of Tougaard is based on the approximate solution for multiple electron scattering in solid [12. This solution assumes differential inelastic scattering cross section of electrons to be depth independent. Accuracy of background subtraction is critically dependent on the right choice of the differential inelastic scattering cross section. Tougaard suggests two kinds of functions (bi-parametric [13. and tri-parametric [14]) for defining of differential inelastic scattering cross section. However, even if it gets possible to make right choice of the parameters, which define differential inelastic scattering cross section in the bulk of solid, the method still suffers from missed account for difference between inelastic scattering in bulk and at the surface. Paper [15] presents Advanced Tougaard Background Method for surfaces, which structure can be presented as a sequence of layers. It is based on representation of the measured spectrum as a superposition of each layer partial spectrum followed by applying Tougaard method to each of them. Problematic though is how to find the partial spectra data. In paper [15, recursive procedure is suggested for that. Provided the right first approximation is found right, the algorithm can demonstrate a reasonable convergence. 
The following formula that accounts for inelastic scattering both in bulk and at the surface (derivation is presented in Appendix Appendix A will be used for calculation of the background in the range of energies between $E_{\min }$ to $E_{\max }$ :

$$
\operatorname{Background}(E)=A \int_{E}^{E_{\max }} j\left(E^{\prime}\right) x_{S B}\left(E-E^{\prime}\right) d E^{\prime}
$$

where $j(E)$ is the measured flux of photoelectrons with energy $E ; A$ is calculated to match background flux at $E_{\min } ; x_{S B}(\Delta)$ is the generalized orthonormalized per unity differential inelastic scattering cross section (further on, orthonormalized per unity differential inelastic scattering cross section will be referred as inelastic indicatrix), $\Delta$ is the energy loss. $x_{S B}(\Delta)$ depends on surface excitation parameter $(S E P)$, as well as on inelastic indicatrices of scattered photoelectrons in the bulk $x_{B}(\Delta)$ and at the surface $x_{S}(\Delta)$ of the sample:

$x_{S B}(\Delta)=x_{B}(\Delta)-\int_{0}^{\Delta} L_{S}(\Delta-\varepsilon) x_{B}(\varepsilon) d \varepsilon+L_{S}(\Delta)$,

where $L_{S}(\Delta)$ is calculated following the formula:

$$
L_{S}(\Delta) \approx \sum_{n=1}^{N}(-1)^{n+1} \frac{S E P^{n}}{n !} y_{n}(\Delta),
$$

$N$ is the maximum accountable multiplicity of inelastic scattering, $y_{n}(\Delta)$ a the multiple inelastic indicatrices, $y_{1}(\Delta)=x_{S}(\Delta), y_{n}(\Delta)=$ $\int_{0}^{\Delta} y_{n-1}(\Delta-\varepsilon) y_{1}(\varepsilon) d \varepsilon$. Parameter SEP depends on energy of photoelectron, composition and surface state. There are different analytic expressions for SEP [16, 17, 18. Chen [16] proposed a simple expression for the $S E P$ for the surface interaction with an electron with energy $E$ at an angle $\theta$ in respect to the surface normal:

$$
S E P=\frac{a_{C H}}{\sqrt{E} \cos \theta}
$$

where $a_{C H}$ is the material dependent parameter.

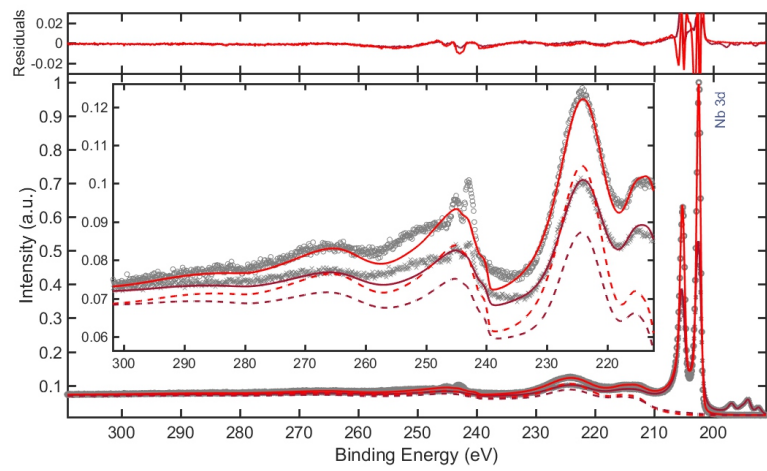

Fig. 1: XPS spectra: line Nb $3 d$. Circles: experimental data [4, $\mathrm{Al}$ anode with a monochromator; criss-crosses: experimental data 4, $\mathrm{Mg}$ anode without a monochromator; solid line: calculation by formulae 1 - 8; dashed line: background calculated by formula $11-5$

Electrons in solid loose energy for ionization, experience strong inelastic scattering due to excitation of surface and bulk plasmons, etc. Differential inelastic scattering cross section can be accounted in the frame of complex dielectric constant formalism [19, 20]. However, for practical calculations, it is more convenient to use more simple approximated forms. Further on, we will use an expression, in which energy losses for excitation of plasma oscillations, as well as losses for ionization of inner electron shells are represented:

$$
x_{i n}(\Delta)=\frac{\sigma_{\mathrm{pl}}}{\sigma_{\text {tot }}} x_{\mathrm{pl}}(\Delta)+\frac{\sigma_{\text {ion }}}{\sigma_{\text {tot }}} x_{\text {ion }}(\Delta),
$$

where $\sigma_{\mathrm{pl}}$ and $\sigma_{\text {ion }}$ are the full cross sections for energy losses due to plasmon excitation and ionization, $\sigma_{\text {tot }}=\sigma_{\mathrm{pl}}+\sigma_{\text {ion }}$ is the full inelastic scattering cross section. We calculate the orthonormalized differential ionization cross section following the formula from paper 21. For calculations of orthonormalized differential cross section for energy loss due to plasmon excitation, we suggest to use formula proved working well for REELS calculations [22, 23]:

$$
x_{\mathrm{pl}}(\Delta)=A_{\text {norm }} \frac{\Delta^{\alpha}}{\left(\Delta^{2}-\varepsilon_{p l}^{2}\right)^{2}+(\gamma \Delta)^{4-\beta}}
$$




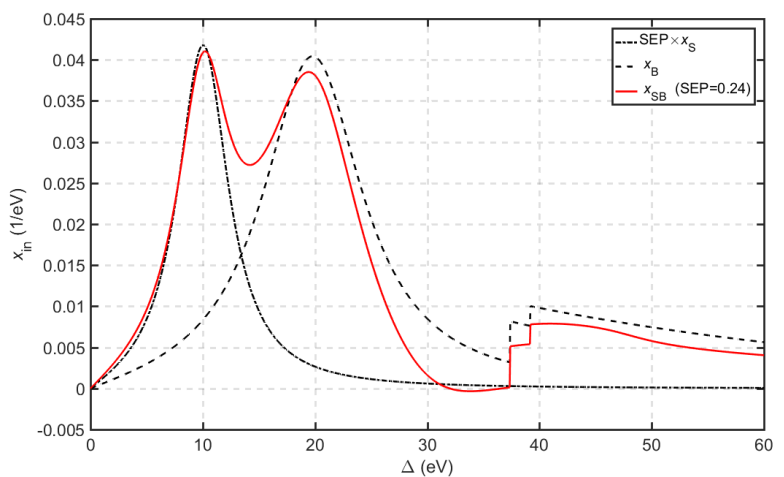

Fig. 2: Inelastic scattering indicatrices. Solid line: the generalized inelastic indicatrix $x_{S B}(\Delta)$ (calculation by formula $(2)$ ); dash-and-dotted line: the inelastic surface indicatrix $x_{S}(\Delta)$ multiplied by the SEP for scaling (calculation by formula $\sqrt{5}$ ) dashed line: the inelastic volume indicatrix $x_{B}(\Delta)$ (calculation by formulae 4 and (5)

where $\varepsilon_{p l}$ is the energy of plasma oscillations, $\alpha, \beta$ are parameters defining the order of $x_{p l}$ dependence on energy loss, $\gamma$ is a parameter, which defines peak width, value of $A_{\text {norm }}$ is defined by normalization condition $\int_{0}^{\infty} x_{\mathrm{pl}}(\varepsilon) d \varepsilon=1$. Applying of $\alpha=1, \beta=2$, $\varepsilon_{p l}=\sqrt{C}, \gamma=2 \sqrt{C}$ to Eq. (5) reduces it to the bi-parametric Tougaard function [13. Applying of $\alpha=1, \beta=2, \varepsilon_{p l}=\sqrt{C}, \gamma=\sqrt{D}$, to Eq. 5 reduces it to the tri-parametric Tougaard function [14].

Figures 1, 2 and 3 show the results of background calculation for spectrum line Nb3d following the presented approach. Circles (Figs. 1 and 3) and crosses (Fig. 1) present experimental data for pure uniform $\mathrm{Nb}$ sample taken from Handbook of X-ray Photoelectron Handbook 4. Circles present experimental data recorded with $\mathrm{X}$-ray source with $\mathrm{Al}$ anode and monochromator, while crosses - with $\mathrm{Mg}$ anode and without monochromator. Dashed line at Fig. 1 shows background calculated following Eq. (1). For calculations, Eq. (2) for generalized inelastic indicatrix was applied. Parameters for background and elastic peaks calculations were found jointly through fitting procedure based on the Marquardt and Levenberg non-linear least squares curve fitting algorithm of 24 . Upper panel of Fig 1 presents difference between experimental and calculated spectra (Residuals) proving excellent agreement between experiment

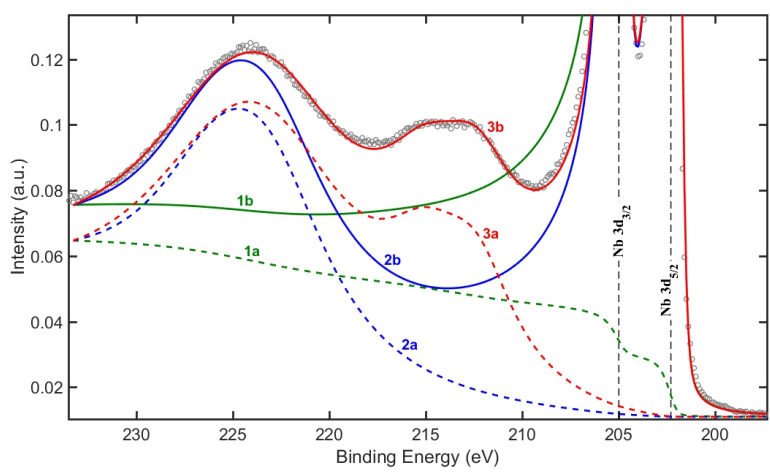

Fig. 3: XPS spectra: line Nb3d. Circles: experimental data [4, $\mathrm{Al}$ anode with a monochromator; solid line (b): calculation by formulae (1) - 8); dashed line (a): background: 1: calculation by the Shirle method, 2 - calculation by the Tougaard method, 3 -calculation by formulae $(1-5)$

and calculations (Adjusted $\mathrm{R}$ squared $R^{2}=0.9945$, mean relative error $2 \%$ ). Method for elastically scattered photoelectrons peaks calculation along with the parameters for the Nb3d line are communicated in the section 3.2 . Figure 2 shows generalized inelastic indicatrix $x_{S B}(\Delta)$ calculated following formula (2) with $\mathrm{SEP}=0.24$ (solid line); inelastic surface scattering indicatrix $x_{S}(\Delta)$ multiplied by $S E P$, calculated following formula (5) with the following parameters $\alpha_{S}=1.10, \beta_{S}=2.08, \varepsilon_{p l S}=10.4 \mathrm{eV}$, $\gamma_{S}=5.5$ (dash-dotted line); inelastic bulk scattering indicatrix $x_{B}(\Delta)$ calculated following the formulas (4) and (5) with the following parameters $\alpha_{B}=1.10, \beta_{B}=2.08, \varepsilon_{p l B}=20.5 \mathrm{eV}, \gamma_{B}=10.5$ ionisation thresholds in Grysinsky formula $J_{1}=37.4 \mathrm{eV}$, $J_{2}=39.2 \mathrm{eV}$ (dashed line). The highest error of the background calculations appears to be for ionization losses. That is due to Grysinsky formula is an approximation. Figure 3 shows comparison of experimental data for the Nb3d line (marked with $\mathrm{b}$ at the plots) and that calculated by different methods (marked with a at the plots): 1 - Shirley method, $2-$ Tougaard method, tri-parametric formula $(C=425$, $D=88$ ), 3 - the presented method, Eqs. (1) - (5). Parameters used for calculation by each method were different and optimized for minimum dispersion following the Marquardt and Levenberg algorithm. It is seen that the presented method gives the best results. 


\subsection{Line Decomposition}

Structure of XPS peaks may be quite complex due to spin-orbit interaction, chemical shift and presence of satellite peaks. Additionally, shape and width of the peaks themselves depend on various factors, and the peaks can overlap each other. For example, Nb3d line may contain up to six doublets (total 12 peaks), as $\mathrm{Nb}$ forms 5 different oxides (maximum oxidation state for niobium $J=5$ ). Accurate decomposition of so complex lines calls for careful account for various factors defining peaks shape and intensity.

Shape of spectral line $L S(E)$ of photoelectrons with kinetic energy $E$ is determined by convolution of functions describing natural shape of the line and its instrumental broadening. Natural shape of the line is defined by Doniach-Sunjic equation 25] $D\left(E, \delta, a_{\mathrm{DS}}\right)$, where $\delta$ is natural width of electron level, $a_{\mathrm{DS}}$ is the Anderson singularity index 26]; if $a_{\mathrm{DS}}=0$, the Doniach-Sunjic equation reduces to Lorentz function. Instrumental broadening follows Gaussian function $G(E, W)$, where $W=$ $\sqrt{W_{s p}^{2}+W_{h \nu}^{2}}, W_{h \nu}$ and $W_{s p}$ are X-ray radiation and instrumental broading linewidths.

$$
L S(E)=\int D\left(E^{\prime}, \delta, a_{\mathrm{DS}}\right) G\left(E-E^{\prime}, W\right) d E^{\prime},
$$

We account for nonmonochromaticity of X-ray source radiation through the following expression:

$$
L S_{1}(E)=L S(E)+\sum_{k=1}^{K} I_{\text {sat } k} L S\left(E+\Delta E_{\text {sat } k}\right),
$$

where $I_{\text {sat }}$ and $\Delta E_{\text {sat }}$ are relative intensities and energy shifts of satellites. These parameters depend on material of X-ray source anode. We use the values of these parameters communicated in [4].

Spectra of photoelectrons of $p-, d-\ldots$ shells have doublet structure due to spin-orbital interaction. Shape of the spin-orbital doublets is described by the following expression:

$$
L S_{2}(E)=\frac{1}{1+\alpha} L S_{1}(E)+\frac{\alpha}{1+\alpha} L S_{1}\left(E-\Delta E_{S O}\right),
$$

where $\alpha$ is the ratio for intensities of spin-orbital splitted lines $(\alpha=1 / 2$ for $p$-shell, and $\alpha=2 / 3$ for $d$ shell), $\Delta E_{S O}$ is the spin-orbital interaction energy.

Line under analysis can consist of doublet of free element, either of doublets of elements in different chemically bonded states. In the latter case, complex line shape is described by expression:

$$
L S_{\Sigma}(E)=I_{0} L S_{2}(E)+\sum_{j=1}^{J} I_{j} L S_{2}\left(E-\Delta E_{C S j}\right) .
$$

where $\Delta E_{C S j}$ is the chemical shift energy, $I_{j}$ is the doublet line intensity, $J$ is the number of the doublet lines.

For accurate decomposition of the complex line depends on the values of the parameters $\delta$ and $a_{\mathrm{DS}}$ and energies $\Delta E_{S O}$ and $\Delta E_{C S}$. Once those are defined, number of variable parameters gets minimal and intensities of the doublet lines can be found by fitting algorithms, e.g. Levenberg-Marquardt.

The natural line width $\delta$ is defined by the lifetime $\tau$ of ionized state formed after photoemission. As a consequence of Heisenberg's uncertainty principle, $\delta \sim \hbar / \tau$. The hole lifetime is defined by radiative recombination process emitting characteristic radiation, or Auger recombination emitting an Augerelectron. Lifetime of vacancy depends on the number of valence electrons $N_{\mathrm{v}}$ [27]. Auger-recombination and photo-fluorescence can be resolved within the same electron shell. Because of that, natural line width may be different for the peaks in a doublet. We propose to approximate $\delta$ with expression:

$$
\delta=\alpha_{s o} \delta_{0}\left(\omega_{f}\left(\frac{N_{\mathrm{v}}}{N_{\mathrm{v} 0}}\right)^{a_{f}}+\omega_{O}\left(\frac{N_{\mathrm{v}}}{N_{\mathrm{v} 0}}\right)^{a_{O}}\right)
$$

where $\delta_{0}$ is the natural line width for free (no chemically bonded) element, $N_{\mathrm{v} 0}$ is the number of valence electrons for free element, $\alpha_{s o}$ is the coefficient accounting for line broadening due to spin-orbital splitting $\left(\alpha_{s o}=1\right.$ for the main peak in a doublet), $\omega_{f}$ is the fluorescence yield, $\omega_{O}=1-\omega_{f}$ is the Auger decay yield. Dependence of fluorescence yield on atomic number is presented in paper [28. Coefficients $a_{f}$ and $a_{O}$ can be found from analysis of experimen- 


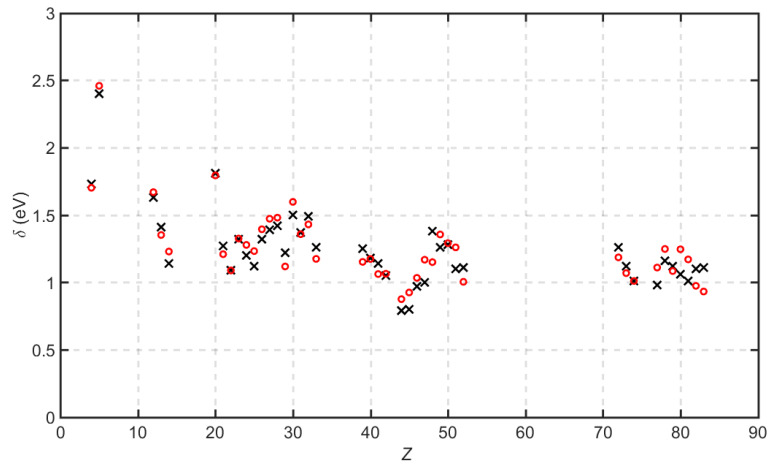

Fig. 4: Dependence of the natural width of the photoelectron oxide line on the atomic number. Circles: experiment [29], criss-crosses: calculation by formula 10

tal data for natural line widths and calculations following formula 10. Figure 4 presents experimental data (circles) for natural line widths of oxides dependence on atomic number $Z$ of elements 29] along with calculations (crosses) by formula with parameters giving the best approximation $\left(a_{f}=0.4305\right.$, $a_{O}=0.5993$ for transition metals, and $a_{f}=0.9444$, $a_{O}=0.5627$ for all others). Mean relative error achieved was $6 \%$.

For pure elements, we suggest taking their natural width $\delta_{0}$, binding energy $B E_{0}$ and spin-orbit interaction energy $\Delta E_{S O}$ from experimental data of Handbook of X-Ray Photoelectron Spectroscopy [4]. Further on, those parameters are kept fixed. For most accurate defining of line parameters for element, we suggest using two sets of of experimental data: spectra recorded with an $\mathrm{X}$-ray source with $\mathrm{Al}$ anode and monochromator, along with those with $\mathrm{Mg}$ anode and without monchromator. Background is suggested to subtract following the approach presented in Section 3.1. Figure 11 presents results calculated with the following Nb3d line parameters: $\delta_{0}=0.0693 \mathrm{eV}$, $\alpha_{s o}=2.30, \alpha_{\mathrm{DS}}=0.136, B E_{0}=202.40 \mathrm{eV}, \Delta E_{S O}=$ $2.72 \mathrm{eV}$. Coefficients $\alpha_{\text {so }}$ and $\alpha_{\mathrm{DS}}$ depend on composition and condition at the surface and are fitting parameters for each sample.

For chemically bonded elements, we suggest calculating the natural line width $\delta_{0}$ following Eq. 10 . Chemical shift energy practically is linearly propor- tional to the oxidation state. For that, it is sufficient to find the chemical shift energy for the highest oxidation state of the element. For example, for niobium oxide, we used $\Delta E_{C S \mathrm{Nb}_{2} \mathrm{O}_{5}}=5.31 \mathrm{eV}$ 30. Section 4 communicates calculation results for the lines following the above presented approach.

\subsection{Extracting of thicknesses of layers}

In paper 31, the formula for calculation of oxide layer thickness is derived for the bi-layer model. In reality however surface always has more complex structure and hence has to be presented as multilayer. In paper [2] , systems of nonlinear equations are presented, which solution gives thicknesses of surface layers. When the number of the layers is large, such calculation scheme starts behaving unstable. In the paper, based on the approach described in [5, 32, 31, the simple formula for extracting of the layers thicknesses is proposed (derivation of that is presented in the Appendix Appendix B):

$$
d_{i}=\lambda_{i} \cos \theta \ln \left(\frac{I_{i} /\left(n_{i} \omega_{p \rightarrow e}(\gamma) \lambda_{i}\right)}{\sum_{j=0}^{i-1} I_{j} /\left(n_{j} \omega_{p \rightarrow e}(\gamma) \lambda_{j}\right)}+1\right)
$$

where $d_{i}$ is the thickness of $i$-th layer, $n$ is the atomic concentration, $\omega_{p \rightarrow e}(\gamma)$ is the differential cross section of photoelectron production [33, $\gamma$ is the angle between the incident radiation and direction to energy analyzer, $\lambda$ is the inelastic mean free path length (IMFP) calculated following TPP2M formula $34, \theta$ is the angle between direction to energy analyzer and surface normal, $I_{i}$ is the intensity of the $i$-th peak. Layer numbering increases from the substrate and up, so that number 0 corresponds to the substrate. In case of bi-layer, Eq. (11) reduces to the formula presented in 31.

To consider elastic scattering effects, IMFP in eq. (11) is to be replaced by the corresponding EAL (Effective-Attenuation-Length):

$d_{i}=L_{i} \cos \theta \ln \left(\frac{I_{i} /\left(n_{i} \omega_{p \rightarrow e i}(\gamma) L_{i}\right)}{\sum_{j=0}^{i-1} I_{j} /\left(n_{j} \omega_{p \rightarrow e j}(\gamma) L_{j}\right)}+1\right)$ 
where $L$ is the average EAL for photoelectrons scattered by the substrate and the film that travel in the film. Values of $L$ can be estimated from a NIST database [35. We use expression derived in work 36 .

$$
L=\lambda\left(1-0.735 \frac{\lambda}{\lambda+\lambda_{t r}}\right)
$$

and $\lambda_{t r}=\frac{1}{n \sigma_{t r}}$ is the transport mean free path (TMFP), $\sigma_{t r}$ is the transport cross section of an elemental solid. Values of transport cross sections are available from the NIST Electron Elastic-Scattering Cross-Section database [37.

For calculating of layer thicknesses in a multilayer sample, assume first that the sample is semi-infinite, metallic and uniform. If oxidation occurred by exposure to oxygen containing gas mixture outside the analysis chamber, then the oxidation states of the surface will decrease from the surface towards interior of the sample [5]. Also, one can expect presence of some hydrocarbons at the very surface, e.g. residuals from the protecting polymer layer (see Experimental section). Calculation of layer thickness will follow a number of consequent steps. First, let assume surface consisting of a number of uniform layers, inside each of which oxidation state is constant. Oxidation state can be not-integer. If in course of calculations thickness of certain layer is found be less than one monolayer (ca. $0.5 \mathrm{~nm}$ ), that is combined with the neighboring one, which further on is considered uniform and multicomponent. Contribution of each layer component is accounted as its relative concentration in the layer. Calculation results for multilayer multicomponent sample is presented in Table 2 of Section 4

\section{Results and Discussion}

In this work, multilayer niobium suboxide films were studied. After loading the sample into the analytic chamber and after each sputtering cycle, XPS survey and detailed spectra of the lines $\mathrm{O} 1 s, \mathrm{C} 1 s$, $\mathrm{Nb} 3 p$ and Nb3d were recorded. Figure 5a shows the survey spectra. Number 0 marks spectra before sputtering, numbers 1,3 and 8 mark spectra after corresponding number of the sputtering cycles. Standard
XPS analysis of the sample before sputtering identified presence of $\mathrm{Nb}, \mathrm{O}$ and $\mathrm{C}$, Fig. 5a. Relative concentrations of those before sputtering were found to be $c_{\mathrm{Nb}}=14 \%, c_{\mathrm{O}}=53 \%$ and $c_{\mathrm{C}}=33 \%$. After the first cycle of sputtering, peaks at the carbon line $\mathrm{C} 1 s$ vanished. Relative concentration of $\mathrm{Nb}$ after each sputtering cycle was as following: $1-47 \%, 2-$ $73 \%, 3-82 \%, 4-82 \%, 5-83 \%, 6-84 \%, 7-93 \%$, $8-96 \%, 9-98 \%$. Abrupt increase of Nb relative concentration after the cycle 7 is due to the change of sputtering cycle parameters (see Section 2).

Standard XPS analysis calculates relative concentrations assuming the sample is uniform at the whole information depth of analysis. However, both before and after sputtering, the sample is nonuniform, multicomponent and multilayer. For quantitative depth profile analysis, recorded lines needs to decomposed into the partial peaks formed by photoelectrons emitted by the different chemically bonded elements. Figs. $5 \mathrm{~b}, 5 \mathrm{c}$ and $5 \mathrm{~d}$ demonstrate experimental spectra and their interpretation following the method presented in the Section 3 . Circles show recorded detailed spectra, the solid lines show calculated spectra. Background removal followed the method presented in the Section 3 . For all calculated peak spectra, the instrumental broadening $W_{s p}=0.575 \mathrm{eV}$ was used, as all the detailed spectra were recorded with the same parameters for energy analyser operated in FAT mode with the pass energy of $20 \mathrm{eV}$. The surface state changed after each sputtering cycle, consequently, so did background parameters. The Doniach-Sunjic asymmetry coefficient $\alpha_{\mathrm{DS}}$ depends on composition and state of surface and thus were the same for each spectral line. This allowed finding the $\alpha_{\mathrm{DS}}$ values for spectra after each sputtering cycle. Linewidths $\delta$ for each peak were calculated following Eq. 10. Details of the spectra before and after cycles 1,3 and 8 of sputtering are summarized in Table 1. In Table 1a the key global, i.e. the common for all spectral peaks, parameters are presented: generalized indicatrix parameters $\left(\varepsilon_{p l B}\right.$ and $\varepsilon_{p l S}$ are the energies of bulk and surface plasmons), $a_{C H}$ parameter for $S E P$ calculation following formula (3) as well as $\alpha_{D S}$ coefficient. Table $1 \mathrm{~b}$ shows, into which peaks the lines are decomposed: binding energy $B E$, chemical 


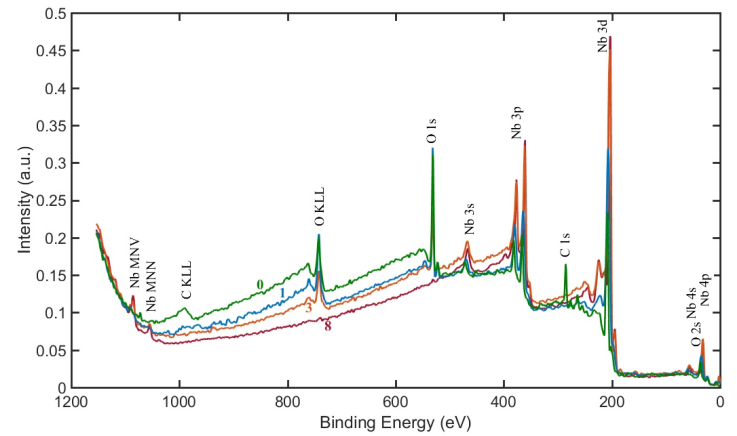

(a)

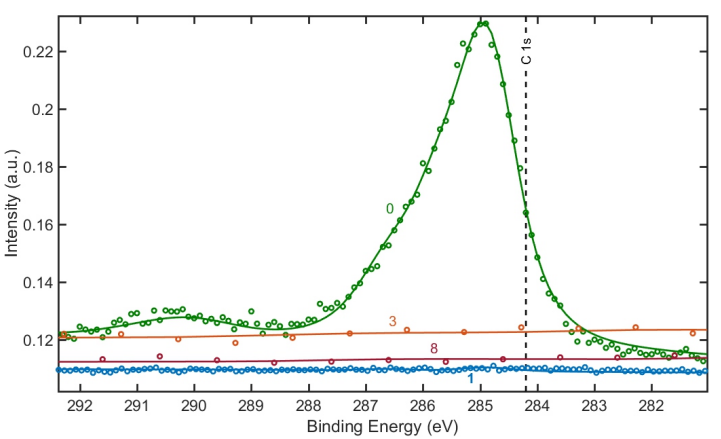

(c)

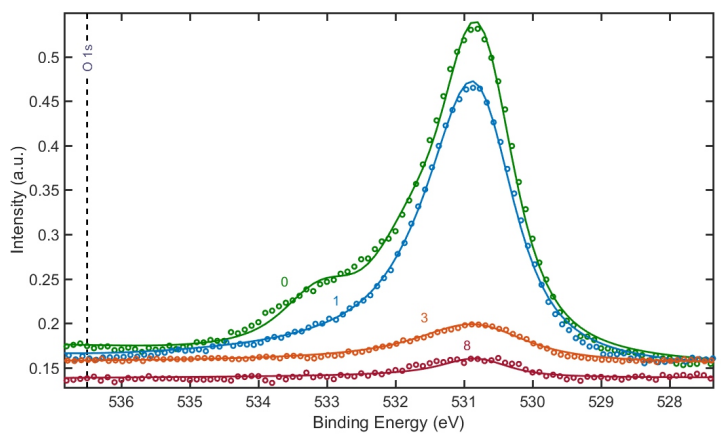

(b)

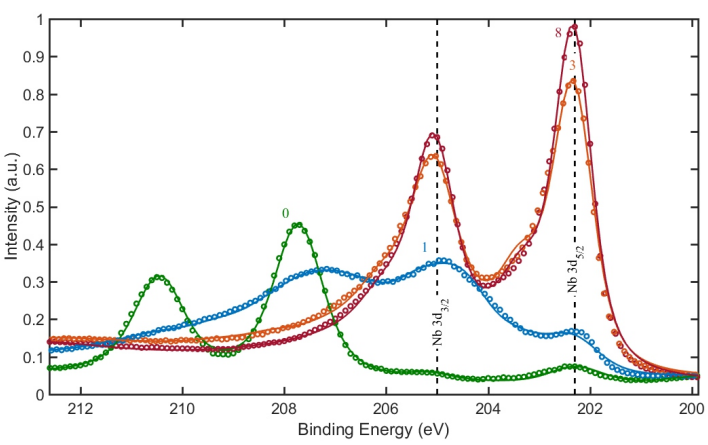

(d)

Fig. 5: XPS spectra. Circles: experimental data, $\mathrm{Mg}$ anode without a monochromator; solid line: calculation by formula 1 (8). The number show sputtering cycles. (a) Target: survey spectra; (b) target: line O $1 s$; (c) target: line C $1 s$; (d) target: line $\mathrm{Nb} 3 d$

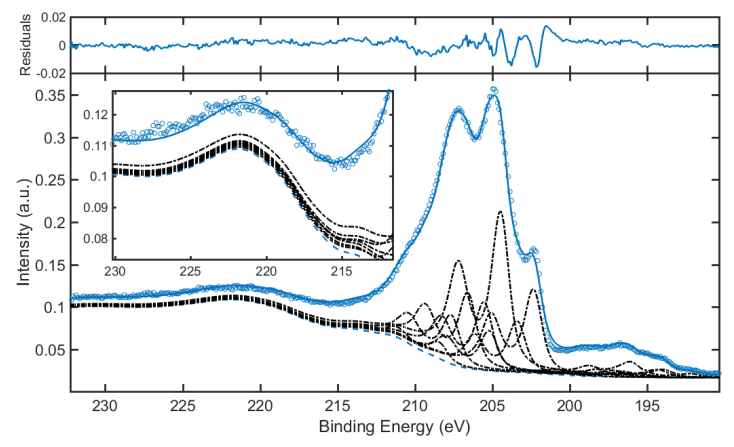

(a)

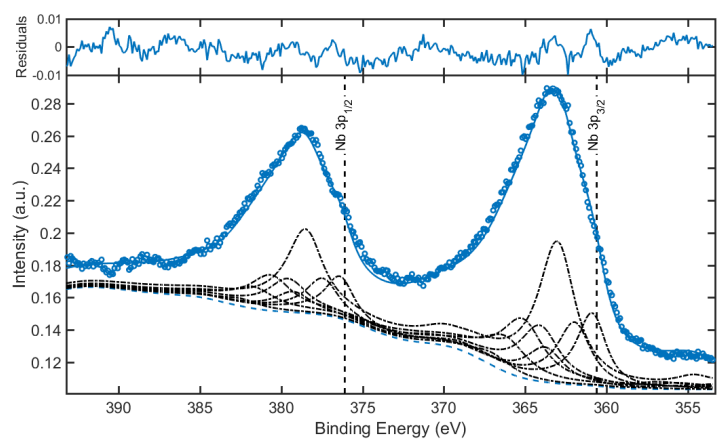

(b)

Fig. 6: XPS spectra. Circles: experimental data, Mg anode without a monochromator; solid line: calculation by formulae 1 8); dashed line (a): background: calculation by formulae (1) - (5); dashed-and-dotted line: separate calculated peaks. (a) Target: line Nb3d; (b) target: line Nb3p 
Table 1: Global and local parameters of line decomposition

(a)

\begin{tabular}{|c|c|c|c|c|c|c|c|c|c|c|c|c|c|c|c|}
\hline \multicolumn{4}{|c|}{0} & \multicolumn{4}{|c|}{1} & \multicolumn{4}{|c|}{3} & \multicolumn{4}{|c|}{8} \\
\hline $\begin{array}{c}\varepsilon_{p l B}, \\
\mathrm{eV}\end{array}$ & $\begin{array}{c}\varepsilon_{p l S} \\
\mathrm{eV}\end{array}$ & $a_{C H}$ & $\alpha_{\mathrm{DS}}$ & $\begin{array}{c}\varepsilon_{p l B} \\
\mathrm{eV}\end{array}$ & $\begin{array}{c}\varepsilon_{p l S} \\
\mathrm{eV}\end{array}$ & $a_{C H}$ & $\alpha_{\mathrm{DS}}$ & $\begin{array}{c}\varepsilon_{p l B} \\
\mathrm{eV}\end{array}$ & $\begin{array}{c}\varepsilon_{p l S} \\
\mathrm{eV}\end{array}$ & $a_{C H}$ & $\alpha_{\mathrm{DS}}$ & $\begin{array}{c}\varepsilon_{p l B} \\
\mathrm{eV}\end{array}$ & $\begin{array}{c}\varepsilon_{p l S} \\
\mathrm{eV}\end{array}$ & $a_{C H}$ & $\alpha_{\mathrm{DS}}$ \\
\hline 24.6 & 14.7 & 19.8 & 0.028 & 26.8 & 13.8 & 23.7 & 0.093 & 20.9 & 10.7 & 10.0 & 0.123 & 20.5 & 10.4 & 7.8 & 0.133 \\
\hline
\end{tabular}

(b)

\begin{tabular}{|c|c|c|c|c|c|c|c|c|c|c|c|c|c|c|c|}
\hline \multicolumn{4}{|c|}{0} & \multicolumn{4}{|c|}{1} & \multicolumn{4}{|c|}{3} & \multicolumn{4}{|c|}{8} \\
\hline $\begin{array}{c}B E \\
\mathrm{eV}\end{array}$ & $\begin{array}{l}c, \\
\%\end{array}$ & $\frac{I}{\sigma_{p} \lambda}$ & Formula & $\begin{array}{c}B E \\
\mathrm{eV}\end{array}$ & $\begin{array}{l}c, \\
\%\end{array}$ & $\frac{I}{\sigma_{p} \lambda}$ & Formula & $\begin{array}{c}B E \\
\mathrm{eV}\end{array}$ & $\begin{array}{l}c, \\
\%\end{array}$ & $\frac{I}{\sigma_{p} \lambda}$ & Formula & $\begin{array}{c}B E \\
\mathrm{eV}\end{array}$ & $\begin{array}{l}c, \\
\%\end{array}$ & $\frac{I}{\sigma_{p} \lambda}$ & Formula \\
\hline \multicolumn{16}{|c|}{$\mathrm{O} 1 \mathrm{~s}$} \\
\hline 530.8 & 73 & 16.32 & $\mathrm{NbO}_{x}$ & 530.8 & 89 & 14.33 & $\mathrm{NbO}_{x}$ & 530.8 & 100 & 4.67 & $\mathrm{NbO}_{x}$ & 530.8 & 100 & 1.18 & $\mathrm{NbO}_{x}$ \\
\hline 531.8 & 16 & 3.66 & $-\mathrm{C}=\mathrm{O}$ & 531.4 & 11 & 1.69 & $\mathrm{Nb}(\mathrm{HO})_{x}$ & & & & & & & & \\
\hline 533.1 & 11 & 2.44 & $-\mathrm{C}-\mathrm{O}$ & & & & & & & & & & & & \\
\hline \multicolumn{16}{|c|}{$\mathrm{C}$ 1s } \\
\hline 284.6 & 64 & 8.76 & $\mathrm{CH}_{x}$ & & & & & & & & & & & & \\
\hline 285.7 & 21 & 2.93 & $-\mathrm{C}-\mathrm{O}$ & & & & & & & & & & & & \\
\hline 286.6 & 15 & 2.05 & $-\mathrm{C}=\mathrm{O}$ & & & & & & & & & & & & \\
\hline \multicolumn{16}{|c|}{$\mathrm{Nb} \mathrm{3d}$} \\
\hline 202.4 & 6 & 0.53 & $\mathrm{Nb}$ & 202.4 & 15 & 2.46 & $\mathrm{Nb}$ & 202.4 & 77 & 19.62 & $\mathrm{Nb}$ & 202.4 & 91 & 22.90 & $\mathrm{Nb}$ \\
\hline 203.4 & 1 & 0.07 & $\mathrm{Nb}_{2} \mathrm{O}$ & 203.4 & 10 & 1.33 & $\mathrm{Nb}_{2} \mathrm{O}$ & 203.4 & 15 & 3.94 & $\mathrm{Nb}_{2} \mathrm{O}$ & 203.4 & 9 & 2.38 & $\mathrm{Nb}_{2} \mathrm{O}$ \\
\hline \multirow[t]{5}{*}{207.7} & 93 & 6.55 & $\mathrm{Nb}_{2} \mathrm{O}_{5}$ & 204.4 & 30 & 4.10 & $\mathrm{NbO}$ & 204.4 & 3 & 0.77 & $\mathrm{NbO}$ & & & & \\
\hline & & & & 205.2 & 10 & 1.44 & $\mathrm{Nb}(\mathrm{HO})_{x}$ & 205.5 & 3 & 0.64 & $\mathrm{Nb}_{2} \mathrm{O}_{3}$ & & & & \\
\hline & & & & 205.5 & 12 & 1.74 & $\mathrm{Nb}_{2} \mathrm{O}_{3}$ & 206.5 & 2 & 0.36 & $\mathrm{NbO}_{2}$ & & & & \\
\hline & & & & 206.5 & 14 & 1.95 & $\mathrm{NbO}_{2}$ & & & & & & & & \\
\hline & & & & 207.7 & 9 & 1.26 & $\mathrm{Nb}_{2} \mathrm{O}_{5}$ & & & & & & & & \\
\hline
\end{tabular}

Table 2: Layer thicknesses based on the XPS results

\begin{tabular}{|c|c|c|c|c|c|c|c|c|c|c|c|}
\hline \multicolumn{3}{|r|}{0} & \multicolumn{3}{|r|}{1} & \multicolumn{3}{|r|}{3} & \multicolumn{3}{|c|}{8} \\
\hline Layer & $\begin{array}{c}d, \\
\mathrm{~nm}\end{array}$ & Formula & Layer & $\begin{array}{c}d, \\
\mathrm{~nm}\end{array}$ & Formula & Layer & $\begin{array}{c}d, \\
\mathrm{~nm}\end{array}$ & Formula & Layer & $\begin{array}{c}d, \\
\mathrm{~nm}\end{array}$ & Formula \\
\hline$\sum$ & 12.0 & & $\sum$ & 6.2 & & $\sum$ & 0.8 & & $\sum$ & 0.6 & \\
\hline 3 & 1.4 & $\begin{array}{c}0.36 \mathrm{C}_{\mathrm{x}}(\mathrm{HO})_{\mathrm{y}}+ \\
0.64 \mathrm{CH}_{\mathrm{x}}\end{array}$ & 4 & 0.5 & $\begin{array}{c}0.31 \mathrm{Nb}(\mathrm{HO})_{\mathrm{x}}+ \\
0.27 \mathrm{Nb}_{2} \mathrm{O}_{5}+ \\
0.42 \mathrm{NbO}_{2}\end{array}$ & 1 & 0.8 & $\begin{array}{c}0.07 \mathrm{NbO}_{2}+ \\
0.13 \mathrm{Nb}_{2} \mathrm{O}_{3}+ \\
0.13 \mathrm{NbO}_{+} \\
0.67 \mathrm{Nb}_{2} \mathrm{O}\end{array}$ & 1 & 0.6 & $\mathrm{Nb}_{2} \mathrm{O}$ \\
\hline 1 & 9.6 & $\mathrm{Nb}_{2} \mathrm{O}_{5}$ & 3 & 1.2 & $\mathrm{Nb}_{2} \mathrm{O}_{3}$ & \multicolumn{2}{|c|}{ Substr. } & $\mathrm{Nb}$ & \multicolumn{2}{|c|}{ Substr. } & $\mathrm{Nb}$ \\
\hline 2 & 1.0 & $\mathrm{Nb}_{2} \mathrm{O}$ & 2 & 1.7 & $\mathrm{NbO}$ & & & & & & \\
\hline \multicolumn{2}{|c|}{ Substr. } & $\mathrm{Nb}$ & 1 & 2.8 & $\mathrm{Nb}_{2} \mathrm{O}$ & & & & & & \\
\hline & & & \multicolumn{2}{|c|}{ Substr. } & $\mathrm{Nb}$ & & & & & & \\
\hline
\end{tabular}


formulas, relative concentration $c$, value $n_{a}=\frac{I}{\sigma_{p} \lambda}$, where $I$ is the intensity of a single or doublet peak, $\sigma_{p}$ is the photoionization cross section for the particular line, $\lambda$ is the IMFP calculated for a particular compound/chemical formula. Parameter $n_{a}$ is proportional to the atomic concentration $n$ of particular element. Calculated for the different lines, but for the same compound chemical formula, $n_{a}$ should be proportional to its stoichiometric coefficients.

For the highest decomposition accuracy, both lines Nb3d and Nb3p. were analysed. For demonstration of the analysis flow, let follow step by step analysis of the most complicated case, surface after the first sputtering cycle. Figure 6 presents detailed spectra of the Nb3d line (Fig. 6a) and Nb3p (Fig. 6b) after the first cycle of sputtering. Dots present the recorded data, solid line is for the calculated spectrum, dashed line shows the background calculated followed the method presented in the Section 3.1, dash-dotted line is for partial doublet peaks. The same background and partial peak parameters (Tabl. 1) were used for calculation of both Nb3d and Nb3p lines.

Parameter $n_{a}$ helps assessing how adequate is decomposition of a spectral line. Let consider a layer containing a number of metal oxides $\mathrm{MO}_{x_{j}}\left(x_{j}=j / 2\right.$, where $\mathrm{j}$ is metal oxidation degree in oxide). Each oxide has a relative concentration $c_{j}$. Then, a mean for the layer stoichiometric coefficient for oxygen is $\langle x\rangle_{c}=\sum_{j} c_{j} \frac{j}{2}$. Suppose, analysis of an XPS line of the metal gave the partial parameters $n_{a j}^{M}$, while analysis of line O1s gave $n_{a}^{O}$. Then mean stoichiometric coefficient for oxygen is $\langle x\rangle_{n}=\frac{n_{a}^{O}}{\sum_{j} n_{a j}^{M}}$, as the $n_{a}$ parameter is proportional to the atomic concentration. Naturally, two ways for stoichiometric coefficient calculation should give the same result. In the other words, that is the criterion for the detailed spectra decomposition into the partial peaks. The following condition should be satisfied:

$$
\sum_{j} c_{j} x_{j}=\frac{n_{a}^{O}}{\sum_{j} n_{a j}^{M}}
$$

It is worth mentioning that the above criterion 14 is satisfied for the presented decomposition into partial peaks (Fig. 5 and Tabl. 1). For example, for the most complicated case of the spectra recorded after first sputtering cycle, the mean stoichiometric coefficient of oxygen, when calculated through the relative concentrations, $\langle x\rangle_{c}=\frac{10 \cdot \frac{1}{2}+30 \cdot \frac{2}{2}+12 \cdot \frac{3}{2}+14 \cdot \frac{4}{2}+9 \cdot \frac{5}{2}}{10+30+12+14+9}=$ 1.3800 , while when calculated through the $n_{a}$ parameter, $\langle x\rangle_{n}=\frac{14.33}{1.33+4.1+1.74+1.95+1.26}=1.3805$.

Decomposition of the detailed spectra into the partial peaks following the approach presented in the Section 3.3 allowed extracting thicknesses of oxide and suboxide layers of niobium. Layer thicknesses were calculated by Eq. (12). Table 2 summarizes the results for chemical and phase depth profile analysis of the surface before and after 1,3 and 8 sputtering cycles.

The probing information depth calculated by the formula 12 for the bilayer model $\left(I_{1} / I_{0}=100 / 1\right)$ is $15 \mathrm{~nm}$.

\section{Conclusions}

In this paper, the new method for XPS chemical and phase depth profile analysis has been presented. The method is based on the most accurate solution of the XPS key problems (see Section 3). It is worth mentioning that the XPS depth profile analysis is a reverse problem with multiple unknown parameters. For the correct solving of the problem, we propose: joint solving of the key problems using the global parameters for all detailed spectra; accounting for different lines of an element for their joint decomposition into partial peaks; decomposition quality assessing with a help of suggested criterion.

\section{Appendix A. Background}

A photoelectron born at a depth $z$ with energy $E_{0}$ needs to travel the length $u$ in the sample's matter before exiting the sample surface at the angle $\theta$ to the surface normal being registered with an energy $E$ by an energy analyzer. On the way, it looses energy $\Delta=E_{0}-E$. Flux density of such electrons is defined by the relation [38, 39]:

$$
q\left(z, \Delta, \theta_{0}, \theta\right)=n \omega_{p \rightarrow e}(\gamma) T_{i n}\left(\Delta, \frac{z}{\cos \theta}\right)
$$


where $n$ is the atomic concentration, $\omega_{p \rightarrow e}$ is the differential photoelectron creation cross section, $\gamma$ is the angle between the direction of photon incidence and travel direction of the created photoelectron; $\lambda=\frac{1}{n \sigma_{i n}}$ IMFP, $\sigma_{i n}$ is the inelastic scattering cross section; $\theta_{0}$ is the angle between the surface normal and direction of photon incidence; $T_{i n}(\Delta, u)$ is the layer's inelastic transmission function, $\Delta$ is the electron energy loss, $u$ is the photoelectron length of travel. For the transmission function, let follow the general solution after Landau [40]:

$$
T_{i n}(\Delta, u)=\frac{1}{2 \pi i} \int_{-i \infty}^{+i \infty} e^{-u n\left(\sigma_{i n}-W(p)\right)} e^{p \Delta} d p
$$

where $W(p)$ is the Laplace image of differential inelastic scattering cross section $\omega_{i n}(\Delta)$. In Laplace space, transmission function has a simple form:

$$
T_{\text {in }}(p, u)=\exp \left(-\frac{u}{\lambda}(1-X(p))\right)
$$

where $X(p)=\frac{W(p)}{\sigma_{i n}}$ is the Laplace image of inelastic indicatrix $x_{i n}(\Delta)=\frac{\omega_{i n}(\Delta)}{\sigma_{i n}}$, which is the orthonormalized differential inelastic scattering cross section. Flux density of all the photoelectrons exiting the sample $Q(\Delta)$ is defined in the Laplace space as following:

$$
\begin{gathered}
Q(p)=\int_{0}^{\infty} n \omega_{p \rightarrow e}(\gamma) e^{-\frac{z}{\lambda \cos \theta}(1-X(p))} d z= \\
\frac{n \omega_{p \rightarrow e}(\gamma) \lambda \cos \theta}{1-X(p)}
\end{gathered}
$$

Let introduce function $\tilde{Q}(p)=\frac{Q(p)}{n \omega_{p \rightarrow e}(\gamma) \lambda \cos \theta}$, then from A.2 follows:

$$
\tilde{Q}(p)-\tilde{Q}(p) X(p)=1
$$

Coming back to the natural space and using the property of Laplace transformation, we get the fol- lowing equation:

$$
\delta(\Delta)=\tilde{Q}(\Delta)-\int_{0}^{\Delta} \tilde{Q}(\varepsilon) x_{i n}(\Delta-\varepsilon) d \varepsilon .
$$

The peak of not scattered and elastically scattered photoelectrons corresponds to the Dirac delta function $\delta(\Delta)$. The convolution integral defines background due to inelastic multiple scattered photoelectrons. $\tilde{Q}(E)$ function is proportional to measured photoelectron flux $j(E)$. Consequently, the Tougaard formula for background 12 can be obtained:

$$
\operatorname{Background}(E)=A \int_{E}^{E_{\max }} j\left(E^{\prime}\right) x_{i n}\left(E-E^{\prime}\right) d E^{\prime},
$$

where $A$ is defined by the spectrum point at the energy $E_{\min }$.

Formula A.5 is obtained with the assumption of the sample uniformity across the sample depth. However, for inelastic scattering real sample is always nonuniform. Electron energy losses at the sample surface and in the bulk follow different relations. As the first approximation, in a semi-infinite sample we select very thin plane-parallel surface layer with the thickness $d_{S}$, where energy losses are defined by surface plasmon excitation. Outside the selected layer, the energy losses are defined by the bulk plasmon excitation and ionization losses. This way, losses in this layer are described by inelastic indicatrix $x_{S}(\Delta)$ (Laplace image $X_{S}(p)$ ), while outside the layer by $x_{B}(\Delta)$ (Laplace image $\left.X_{B}(p)\right)$. The IMFP in this layer is designated as $\lambda_{S}$, outside the layer $\lambda_{B}$. Then, the Laplace image of transmission function for the photoelectrons passing a layer belonging to the bulk of the sample with thickness $z$ and a thin layer with thickness $d_{S}$ at the angle $\theta$ to the surface normal is defined as:

$$
T_{i n}(p, u)=e^{-\frac{z}{\lambda_{B} \cos \theta}\left(1-X_{B}(p)\right)} e^{-\frac{d_{S}}{\lambda_{S} \cos \theta}\left(1-X_{S}(p)\right)} .
$$


Surface excitation parameter $(S E P)$ can be represented as:

$$
S E P=\frac{d_{S}}{\lambda_{S} \cos \theta} .
$$

IMFP of photoelectrons is proportional to their energy, $\lambda \sim E^{a}$. Parameter a is usually within the range of $0.5 \ldots 0.9$. With $a=0.5$ A.7) gets reduced to the Chen formula [16. Plugging (A.6) to A.1) and integrating across the whole sample thickness and accounting for A.7) Laplace image of the full flux density of all photoelectrons escaping the sample surface:

$$
Q_{S B}(p)=\frac{n \omega_{p \rightarrow e}(\gamma) \lambda_{B} \cos \theta}{1-X_{B}(p)} e^{-S E P\left(1-X_{S}(p)\right)} .
$$

Introducing the functions:

$$
\begin{array}{r}
X_{S B}(p)=1-e^{-S E P X_{S}(p)}\left(1-X_{B}(p)\right), \\
Q_{S B}^{\tilde{A}}(p)=\frac{Q_{S B}(p)}{n \omega_{p \rightarrow e}(\gamma) \lambda_{B} \cos \theta e^{-S E P}}
\end{array}
$$

a relation similar to A.3 is obtained:

$$
\tilde{Q}_{S B}(p)-\tilde{Q}_{S B}(p) X_{S B}(p)=1 .
$$

Similarly as for deriving (A.5), the background calculation formula accounting for inelastic scattering at the surface and in bulk is obtained:

$$
\operatorname{Background}(E)=A \int_{E}^{E_{\max }} j\left(E^{\prime}\right) x_{S B}\left(E-E^{\prime}\right) d E^{\prime} .
$$

Fundamental difference between Eqs. A.5 and A.11 though is that the function under integral $x_{S B}(\Delta)$ in (A.11) depends on $S E P$, as well as inelastic photoelectron scattering indicatrix in bulk $x_{B}(\Delta)$ and at the surface $x_{S}(\Delta)$. For deriving of the calculation formula for $x_{S B}(\Delta)$, let re-write Eq. A.9 in the following form:

$$
X_{S B}(p)=X_{B}(p)-X_{B}(p) L(p)+L(p),
$$

where $L(p)=1-e^{-S E P X_{S}(p)} \cdot L(p)$ function can be expanded in a power series $L(p)=\sum_{n=1}^{\infty}(-1)^{n+1} \frac{S E P^{n}}{n !} X_{S}^{n}(p)$. After reversed Laplace transformation the obtained formula for $x_{S B}(\Delta)$ are the following:

$x_{S B}(\Delta)=x_{B}(\Delta)-\int_{0}^{\Delta} L_{S}(\Delta-\varepsilon) x_{B}(\varepsilon) d \varepsilon+L_{S}(\Delta)$

where $L_{S}(\Delta) \approx \sum_{n=1}^{N}(-1)^{n} \frac{S E P^{n}}{n !} y_{n}(\Delta)$, $y_{1}(\Delta)=x_{S}(\Delta), y_{n}(\Delta)=\int_{0}^{\Delta} y_{n-1}(\Delta-\varepsilon) y_{1}(\varepsilon) d \varepsilon$, $N$ is the maximum accountable multiplicity of inelastic scattering.

\section{Appendix B. Layer Thickness}

The method for calculation of the layers thicknesses in multilayer model is based on the approach presented in the papers [5, 32, 31]. The flux of photoelectrons born at the depth $z$ with the energy $E_{0}$ loselessly escaping the sample surface at the angle $\theta$ to its normal is defined by the following formula:

$$
q\left(z, \theta_{0}, \theta\right)=n \omega_{p \rightarrow e}(\gamma) \exp \left(-\frac{z}{\lambda \cos \theta}\right)
$$

where $n$ is the atomic concentration, $\omega_{p \rightarrow e}$ is the differential electron creation cross section, $\lambda$ is the photoelectron's IMFP, $\gamma$ is the scattering angle. For defining of flux density of the photoelectrons escaping a uniform layer with a depth $d_{i}$, integrating of Eq. (B.1) across the whole layer thickness is needed:

$$
Q\left(d_{i}, \theta_{0}, \theta\right)=\int_{0}^{d_{i}} q\left(z, \theta_{0}, \theta\right) d z .
$$

After integration, we obtain:

$$
Q\left(d_{i}, \theta_{0}, \theta\right)=n_{i} \omega_{p \rightarrow e i}(\gamma) \lambda_{i} \cos \theta\left(1-e^{-\frac{d_{i}}{\lambda_{i} \cos \theta}}\right)
$$


Designating $T_{i}=\exp \left(-\frac{d_{i}}{\lambda_{i} \cos \theta}\right), \quad Q_{i}^{\infty}=$ $Q_{i}\left(\infty, \theta_{0}, \theta\right)=n_{i} \omega_{p \rightarrow e}(\gamma) \lambda_{i} \cos \theta$ allows rewriting Eq. B.3 in a compact form:

$$
Q_{i}=Q_{i}^{\infty}\left(1-T_{i}\right)
$$

is proportional to the measured peak intensity $I_{i}$. Let a sample consist of $N$ layers over a substrate with each $i$-th layer having $d_{i}$ thickness. Numbering of the layers does from the substrate and up (the substrate gets number 0 ). The flux density of photoelectrons born within the $i$-th layer and passed the layers with numbers from $i+1$ to $N$ is the following:

$$
Q_{i, \ldots, N}=Q_{i}^{\infty}\left(1-T_{i}\right) T_{i+1} \cdot \ldots \cdot T_{N}
$$

For all layers and the substrate, the following system of non-linear equations is obtained:

$$
\begin{aligned}
& Q_{0, \ldots, N}=Q_{0}^{\infty} T_{1} \cdot \ldots \cdot T_{N} \\
& Q_{1, \ldots, N}=Q_{1}^{\infty}\left(1-T_{1}\right) T_{2} \cdot \ldots \cdot T_{N} \\
& \ldots \\
& Q_{i, \ldots, N}=Q_{i}^{\infty}\left(1-T_{i}\right) T_{i+1} \cdot \ldots \cdot T_{N} \\
& \ldots \\
& Q_{N}=Q_{N}^{\infty}\left(1-T_{N}\right)
\end{aligned}
$$

From the system, thicknesses $d_{i}$ of the layers can be found. For that let us divide the flux density $Q_{i, \ldots, N}$ of photoelectrons generated in the layer $i$ to the flux density $Q_{0, \ldots, N}$ of photoelectrons generated in the substrate. This relation will be equal to the relation of the corresponding peak intensities

$$
\frac{I_{i}}{I_{0}}=\frac{Q_{i}^{\infty}}{Q_{0}^{\infty}} \frac{\left(1-T_{i}\right)}{T_{1} \cdot \ldots \cdot T_{i}}
$$

Let us introduce $f_{i}=I_{i} Q_{0}^{\infty} /\left(Q_{i}^{\infty} I_{0}\right)$. Then expression (B.4) transforms into an iterative formula

$$
\frac{1}{T_{i}}=f_{i} \cdot T_{1} \cdot \ldots \cdot T_{i-1}+1
$$

from that an expression for $T_{i}$

$$
\frac{1}{T_{i}}=\frac{f_{i}}{f_{i-1}+\ldots+f_{1}+1}+1
$$

may be derived. Next, using the introduced designations and functions, we get a simple formula for layer thicknesses

$$
d_{i}=\lambda_{i} \cos \theta \ln \left(\frac{I_{i} /\left(n_{i} \omega_{p \rightarrow e i}(\gamma) \lambda_{i}\right)}{\sum_{j=0}^{i-1} I_{j} /\left(n_{j} \omega_{p \rightarrow e j}(\gamma) \lambda_{j}\right)}+1\right)
$$

\section{References}

[1] C. Powell, New data resources and applications for aes and xps, Journal of Surface Analysis 20 (3) (2014) 155-160.

[2] C. Olsson, D. Landolt, Atmospheric oxidation of a Nb-Zr alloy studied with XPS, Corrosion Science 46 (1) (2004) 213-224. doi:10.1016/ S0010-938X (03) 00139-2.

[3] A. Trifonov, A. Lubenchenko, V. Polkin, A. Pavolotsky, S. Ketov, D. Louzguine-Luzgin, Difference in charge transport properties of ni-nb thin films with native and artificial oxide, Journal of Applied Physics 117 (12) (2015) 125704.

[4] J. Chastain, R. C. King, J. Moulder, Handbook of X-ray photoelectron spectroscopy: a reference book of standard spectra for identification and interpretation of XPS data, Physical Electronics Eden Prairie, MN, 1995.

[5] M. Grundner, J. Halbritter, Xps and aes studies on oxide growth and oxide coatings on niobium, Journal of Applied Physics 51 (1) (1980) 397405 .

[6] A. Darlinski, On angle resolved x-ray photoelectron spectroscopy of oxides, serrations, and protusions at interfaces, Journal of Vacuum Science \& Technology A: Vacuum, Surfaces, and Films 5 (4) (1987) 1235. doi:10.1116/1.574779.

[7] J. Halbritter, On the Oxidation and on the Superconductivity of Niobium, Applied Physics A 43 (1987) (1987) 1-28. doi:10.1007/ BF00615201. 
[8] M. Kuznetsov, A. Razinkin, A. Ivanovskii, Oxide nanostructures on a nb surface and related systems: experiments and ab initio calculations, Physics-Uspekhi 53 (10) (2010) 995.

[9] Z. Weibin, W. Weidong, W. Xueming, C. Xinlu, Y. Dawei, S. Changle, P. Liping, W. Yuying, B. Li, The investigation of nbo2 and nb2o5 electronic structure by xps, ups and first principles methods, Surface and Interface Analysis 45 (8) (2013) 1206-1210.

[10] D. A. Shirley, High-resolution x-ray photoemission spectrum of the valence bands of gold, Physical Review B 5 (12) (1972) 4709.

[11] S. Tougaard, Practical algorithm for background subtraction, Surface Science Letters 216 (3) (1989) A330. doi:10.1016/0167-2584(89) 91270-X.

[12] S. Tougaard, I. Chorkendorff, Differential inelastic electron scattering cross sections from experimental reflection electron-energy-loss spectra: Application to background removal in electron spectroscopy, Physical Review B 35 (13) (1987) 6570-6577. doi:10.1103/PhysRevB.35.6570.

[13] S. Tougaard, Low energy inelastic electron scattering properties of noble and transition metals, Solid State Communications 61 (9) (1987) 547549. doi:10.1016/0038-1098(87)90166-9.

[14] S. Tougaard, Universality Classes of Inelastic Electron Scattering Crosssections, Surface and Interface Analysis 25 (November 1996) (1997) 137-154. doi :10.1002/(SICI) 1096-9918(199703)25: 3<137: :AID-SIA230>3.0.CO;2-L

[15] R. Hesse, M. Weiß, R. Szargan, P. Streubel, R. Denecke, Improved peak-fit procedure for XPS measurements of inhomogeneous samples - Development of the advanced Tougaard background method, Journal of Electron Spectroscopy and Related Phenomena 205 (2015) 2951. doi:10.1016/j.elspec.2015.06.013. URL http://dx.doi.org/10.1016/j.elspec. 2015.06 .013
[16] Y. Chen, Surface effects on angular distributions in X-ray-photoelectron spectroscopy, Surface Science 519 (1-2) (2002) 115-124. doi: 10.1016/S0039-6028(02)02206-9

[17] K. Salma, Z. Ding, H. Li, Z. Zhang, Surface excitation probabilities in surface electron spectroscopies, Surface science 600 (7) (2006) 15261539.

[18] W. S. Werner, L. Kövér, S. Egri, J. Toth, D. Varga, Measurement of the surface excitation probability of medium energy electrons reflected from si, ni, ge and ag surfaces, Surface science 585 (1) (2005) 85-94.

[19] J. Lindhard, On the properties of a gas of charged particles mat. fys. medd, K. Dan. Vid. Sel 28 (8).

[20] R. Ritchie, A. Howie, Electron excitation and the optical potential in electron microscopy, Philosophical Magazine 36 (2) (1977) 463-481.

[21] M. Gryziński, Classical theory of atomic collisions. i. theory of inelastic collisions, Physical Review 138 (2A) (1965) A336.

[22] V. Afanas'ev, A. Lubenchenko, M. Gubkin, Quantitative interpretation of eels and reels spectra, The European Physical Journal BCondensed Matter and Complex Systems 37 (1) (2004) 117-125.

[23] V. Afanasyev, D. Efremenko, A. Lubenchenko, M. Vos, M. Went, Extraction of cross-sections of inelastic scattering from energy spectra of reflected atomic particles, Bulletin of the Russian Academy of Sciences: Physics 74 (2) (2010) 170174.

[24] J. J. Moré, The levenberg-marquardt algorithm: implementation and theory, in: Numerical analysis, Springer, 1978, pp. 105-116.

[25] S. Doniach, M. Sunjic, Many-electron singularity in x-ray photoemission and x-ray line spectra from metals, Journal of Physics C: Solid State Physics 3 (2) (1970) 285. 
[26] P. Anderson, Infrared catastrophe in fermi gases with local scattering potentials, Physical Review Letters 18 (24) (1967) 1049.

[27] D. Spanjaard, C. Guillot, M.-C. Desjonqueres, G. Tréglia, J. Lecante, Surface core level spectroscopy of transition metals: A new tool for the determination of their surface structure, Surface Science Reports 5 (1) (1985) 1-85.

[28] M. Chen, H. Crasemann, B.and Mark, Relativistic $\mathrm{m}$-shell radiationless transitions, Physical Review A 21 (2) (1980) 449.

[29] J. Shea, Handbook of monochromatic XPS spectra - The elements and native oxides [Book Review], IEEE Electrical Insulation Magazine 19 (4). doi:10.1109/MEI.2003.1226740

[30] A. V. Naumkin, A. Kraut-Vass, C. Powell, NIST X-ray photoelectron spectroscopy database, Measurement Services Division of the National Institute of Standards and Technology (NIST) Technology Services, 2008.

[31] B. Strohmeier, An esca method for determining the oxide thickness on aluminum alloys, Surface and Interface Analysis 15 (1) (1990) 51-56.

[32] T. Carlson, Basic assumptions and recent developments in quantitative xps, Surface and Interface Analysis 4 (4) (1982) 125-134.

[33] J. Yeh, I. Lindau, Atomic subshell photoionization cross sections and asymmetry parameters: 1; zi 103, Atomic data and nuclear data tables 32 (1) (1985) 1-155.

[34] S. Tanuma, C. Powell, D. Penn, Calculations of electron inelastic mean free paths. v. data for 14 organic compounds over the 50-2000 ev range, Surface and Interface Analysis 21 (3) (1994) $165-176$.

[35] C. J. Powell, A. Jablonski, NIST Electron Effective-Attenuation-Length Database, Version 1.3 U.S. Department of Commerce/NIST: Gaithersburg, MD, 2011.

URL https://www.nist.gov/srd/ nist-standard-reference-database-82
[36] A. Jablonski, C. Powell, Practical expressions for the mean escape depth, the information depth, and the effective attenuation length in auger-electron spectroscopy and x-ray photoelectron spectroscopy, Journal of Vacuum Science \& Technology A 27 (2) (2009) 253-261.

[37] A. Jablonski, F. Salvat, C. Powell, NIST Electron-Scattering Cross-Section Database, Version 3.2, U.S. Department of Commerce/NIST: Gaithersburg, MD, 2010.

URL https://www.nist.gov/srd/ database-64

[38] S. Tougaard, P. Sigmund, Influence of elastic and inelastic scattering on energy spectra of electrons emitted from solids, Physical Review B 25 (7) (1982) 4452.

[39] S. Tougaard, Energy loss in xps: Fundamental processes and applications for quantification, non-destructive depth profiling and 3d imaging, Journal of Electron Spectroscopy and Related Phenomena 178 (2010) 128-153.

[40] L. Landau, On the energy loss of fast particles by ionization, Originally published in J. Phys 8 (1944) 201. 